\title{
Editorial
}

\section{Elimination of Lymphatic Filariasis from Bangladesh: Current Status}

\author{
Md. Moazzem Hossain ${ }^{1}$
}

Bangladesh is a developing country with lots of communicable diseases. Among those communicable diseases majority are prevented by vaccination program. Lymphatic filariasis (LF) is one of the communicable diseases which cannot be prevented by vaccination. For prevention of this disease elimination is the mainstay.

Bangladesh is situated in the South-East Asian region with an estimated population of 142.30 million. It is one of the most populous LF endemic countries. The disease is caused by the parasite Wuchereria bancrofti; Culex mosquitoes are the main vectors for transmission. In 2001, an assessment based on microfilaria (Mf) levels found the infection in 34 of the 64 districts with 19 districts eligible for mass drug administration (MDA). It has been estimated that a population of 69 million people are living in endemic zones and the highest rates of infection and disease are in the northern part of the country where up to $16.8 \%$ of the population is Mf-positive and 10.1\% have chronic disease (Hafiz 2012).

Over the past decade it has made in a great progress in the context of the elimination of LF. Bangladesh is one of the first countries to start the elimination programme by adopting mass drug administration (MDA) strategy with the support of the Global Programme to Eliminate LF (GPELF). Currently it is one of the first country which is going to begin the verification process using the new WHO guidelines of the Transmission Assessment Survey (TAS). In 2010, 13 out of 19 districts had completed five or more rounds of MDA; however, Mf prevalence rate was found to be zero in 5 districts according to Mf sentinel surveys (Hafiz 2012). These five districts have therefore been targeted to verify the interruption of transmission using the new, transmission assessment survey (TAS). The TAS was a big challenge for the program due to resource and staff constraints. Involving primary health care staff made the survey a cost effective and successful. To date, the TAS has been completed in 5 districts with great success as ICT positive in children were found to be lower than cut off point; thus confirming the interruption of transmission. It is important to mention here that Bangladesh was one of the first countries to use the new TAS protocol developed by WHO in collaboration other international institutions. Based on the TAS results MDA has now stopped in five districts that were previously highly endemic. This is an incredible achievement and the PELF plans to conduct TAS in a further five districts, which was completed at the end of 2011. It is anticipated that transmission will also be interrupted in these districts, indicating that the Bangladesh PELF is well on its ways towards elimination in spite of high density of population and limited resources.

There has been an incredibly strong commitment from the Bangladesh Government, Ministry of Health and Family Welfare (MOHFW) over the past decade. This commitment together with the support of external donors/stakeholders has made it possible to achieve the many things in the programme. The main donors have been the Centre for Neglected Tropical Diseases (CNTD) Liverpool, USAID, GlaxoSmithKline (GSK), JICA, RTI, LF Support Centre, Atlanta USA. It has truly been an international collaborative effort and the Bangladesh people and Government are thankful for the support.

Bangladesh expects to eradicate lymphatic filariasis over the next two years. The disease causes swelling of parts of human body, with health officials saying their campaign so far succeeded in eradicating the menace from 18 out of 19 hyper endemic districts (Hafiz 2012). The disease still persists in few zones. It may take

\footnotetext{
${ }^{1}$ Professor of Microbiology \& Chairman, Institute of Allergy and Clinical Immunology of Bangladesh (IACIB), Savar, Dhaka \& Former Director, Communicable Disease Control, Directorate General of Health Services, Ministry of Health and Family Welfare, Mohakhali, Dhaka, Bangladesh \& Founder President, Filaria \& General Hospital, Savar, Dhaka, Bangladesh; Email: moazzem.iacib@gmail.com ; Cell no.: +8801715566084
} 
few months to year to wipe out it from there. Communicable disease control wing of Directorate General of Health Services of MOHFW has been started this elimination program. Filariasis Hospital in suburban Savar has played a key role in the research field related to filariasis elimination from non-government side. Bangladesh launched the National Filariasis Elimination Programme in 2001 with an aim to eliminate it by 2015 in response to the World Health Organization (WHO) that has launched global programme to wipe out Lymphatic Filariasis (GPELF) by 2020 (WHO 2016).

Fund shortage under this programme caused a little setback so far; however, it can still possible to eliminate filariasis far ahead of the WHO's fixed time of 2020. According to a nationwide survey in 2005 with Japanese assistance found two million people to be victims of filariasis in Bangladesh and it should conduct another survey to know the present status of the filariasis patients.

[Journal of Science Foundation 2016;14(1):1-2]

\section{References}

Hafiz I. Final Abstract Number: 11.002 Session: NTDs in Asia and Pacific: Progress towards Elimination and Control Date: Thursday, June 14, 2012 Time: 15:45-17:45 Room: Ballroom C Elimination of lymphatic filariasis- Bangladesh experience Filariasis Elimination Program, Ministry of Health \& Family Welfare, Dhaka, Bangladesh

World Health Organization (WHO). 2016. Neglected Tropical Disease; Neglected Tropical Disease (Kala-zar, Filariasis and Dengue); Web Address: http://www.searo.who.int/bangladesh/areas/tropical_disease/en/\# 